\title{
Pleural Effusions, Eosinophilia and a Positive Interferon- $\gamma$ Release Assay
}

\author{
Jessica Howard-Andersonc Timothy Canan ${ }^{c}$ Michael D. Roth ${ }^{\mathrm{b}, \mathrm{c}}$ Edward Haa, c \\ Divisions of a General Internal Medicine and ${ }^{b}$ Pulmonary and Critical Care, ${ }^{C}$ Department of Medicine, UCLA Medical \\ Center, Los Angeles, Calif., USA
}

\section{Case Report}

A 91-year-old Korean male with a history of hepatitis C liver cirrhosis with portal hypertension, irritable bowel syndrome and chronic gastritis presented to the hospital with fever for 2 days, productive cough and hemoptysis. His history also included two admissions to outside hospitals in the preceding year for presumed bacterial pneumonia and chronic left loculated pleural effusion (PE) discovered 4 months prior to his presentation. A thoracentesis at the outside hospital had revealed an exudative process with $21 \%$ eosinophils. At that time, a pigtail catheter was placed without improvement or re-expansion of the lung, but definitive video-assisted thoracoscopic surgery was not recommended due to the patient's comorbidities. The patient was left with a partial collapse of the left lung, persistent left-sided chest pressure and a chronic mild dry cough. During the week prior to hospital admission, an outpatient pulmonologist sent a Mycobacterium tuberculosis QuantiFERON ${ }^{\circledR}$-TB Gold In-Tube interferon- $\gamma$ release assay (IGRA) for sensitization against M. tuberculosis-specific antigens, which returned strongly positive. Based on this result and the development of fever and hemoptysis, the patient was admitted for further evaluation. A complete history was otherwise nega- tive except for a 1-kg weight loss in the preceding 4 months. His social history was notable for living in South Korea his entire life until immigrating to the United States 10 years prior to his present illness, with no intercurrent travel since that time.

On physical examination, his vital signs were notable for a temperature of $37.9^{\circ} \mathrm{C}$ with normal respiratory rate and pulse oximetry on room air. Pulmonary examination revealed left basal crackles and decreased breath sounds in the left middle and lower lung fields. Admission laboratory data were significant for a white blood cell count of $6.39 \times 10^{3} / \mu \mathrm{l}$, a differential count showing $46 \%$ neutrophils, $24 \%$ lymphocytes, $7 \%$ monocytes and $21 \%$ eosinophils (absolute eosinophil count $1.4 \times 10^{3} / \mu \mathrm{l}$ ) and a platelet count of $90 \times 10^{3} / \mu \mathrm{l}$. His IgE level was elevated at 1.46 $\times 10^{3} \mu \mathrm{g} / \mathrm{l}$. On review of prior laboratory results, he had similar peripheral eosinophilia and elevated IgE level 4 years earlier. All other laboratory data were unremarkable, including a negative enzyme immunoassay (EIA) for Histoplasma urine antigen, Aspergillus antigen, and Coccidioides and HIV antibodies. Tests for C- and PANCAs and stool ova and parasites were also negative.

A chest CT identified a complex loculated left PE with left lung volume loss. In addition, there were groundglass and airspace opacities in the right upper and mid-

\section{KARGER}

E-Mail karger@karger.com

www.karger.com/res
2013 S. Karger AG Basel

0025-7931/13/0866-0508\$38.00/0
Assist. Prof. Edward Ha, MD

Department of Medicine, UCLA Medical Center

757 Westwood Plaza, Suite 7501K

Los Angeles, CA 90095 (USA)

E-Mail Eha@mednet.ucla.edu 
Fig. 1. A high-resolution CT scan of the chest $(\mathbf{a}, \mathbf{b})$ demonstrated a complex loculated left PE with adjacent pleural thickening, airway inflammation and scarring, and volume loss. Multifocal ground-glass and airspace opacities were identified in the posterior right upper and middle lobes along with bronchial wall thickening. Incidental findings included a noncalcified granuloma in the right apex, a hiatal hernia and a markedly patulous esophagus. Concurrent pulmonary function testing (c) confirmed a moderate restrictive process with concurrent mild airway obstruction and a reduced diffusing capacity measurement.
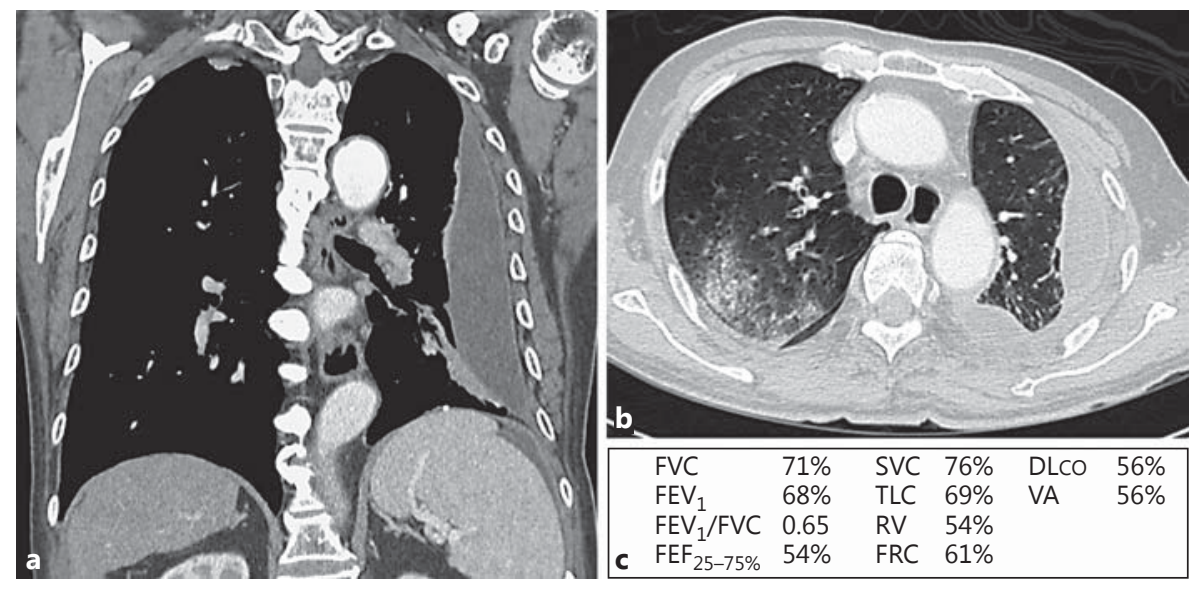

dle lobes with adjacent bronchial wall thickening. A noncalcified granuloma was noted in the right apex (fig. 1).

Bronchoalveolar lavage was performed with cultures positive for rare methicillin-sensitive Staphylococcus aureus. Acid-fast stain (and eventually culture), M. tuberculosis PCR, and testing for ova and parasites were negative. A diagnostic thoracentesis yielded $150 \mathrm{ml}$ of bloody fluid with 264,000 red blood cells $/ \mathrm{mm}^{3}$ and 44 white blood cells $/ \mathrm{mm}^{3}$ with $24 \%$ eosinophils. Pleural fluid total protein was $21 \mathrm{~g} / \mathrm{l}$ and lactate dehydrogenase was $752 \mathrm{U} / \mathrm{l}$. His serum total protein was $77 \mathrm{~g} / \mathrm{l}$ and serum lactate dehydrogenase was $188 \mathrm{U} / \mathrm{l}$. Adenosine deaminase was $6.0 \mathrm{U} / \mathrm{l}$. The pleural fluid was consistent with an exudative process by Light's criteria. Wet preparation of the pleural fluid was negative for ova or parasites. All cultures were negative and no malignant cells were identified.

What Is Your Diagnosis? 


\section{Diagnosis: Strongyloides stercoralis Infection with Pulmonary Involvement}

This patient's initial problem was that of an eosinophilic PE defined as $\geq 10 \%$ eosinophils in the pleural fluid. A meta-analysis published in 2012 on 687 cases of eosinophilic PE found malignancy to be the most common cause (26\%), followed by idiopathic causes (25\%) and parapneumonic effusions (13\%). Other rarer causes for eosinophilic PE, including but not limited to tuberculosis, collagen vascular diseases, pulmonary embolus and gastrointestinal disorders, were also identified [1]. Given our patient's hemoptysis, recent positive QuantiFERON-TB Gold InTube IGRA, radiographic evidence of granulomas and presentation with fever, our suspicion for active pulmonary tuberculosis was initially high. However, sputum cultures, bronchoalveolar lavage and thoracentesis cultures were all negative for acid-fast bacteria. In addition, the pleural fluid adenosine deaminase level was not elevated, essentially ruling out tuberculous effusion as the diagnosis.

On the day of discharge, a second stool sample was tested for ova and parasites and revealed S. stercoralis rhabditiform larvae. Serum EIA testing for Strongyloides antibody also eventually returned positive at 8.36 (reference range $<1$ ), confirming the diagnosis. The positive QuantiFERON-TB Gold In-Tube IGRA in our patient likely reflects latent tuberculosis infection given the patient's prior long-term residence in an endemic area and the presence of a noncalcified granuloma on CT imaging. Additionally, there have been no reports of cross-reactivity between this IGRA assay and parasitic diseases.

S. stercoralis is a nematode that is estimated to infect $30-100$ million people around the world. It is most common in developing nations, particularly Southeast Asia, sub-Saharan Africa and Latin America [2]. The nematode has both a rhabditiform and filariform phase. Filariform larvae are infectious and can penetrate the skin, migrating through the circulatory system to the lungs. After penetrating the alveoli, they ascend the respiratory tract, are subsequently swallowed and reside in the small intestine. There they lay eggs that hatch primarily into rhabditiform larvae and are excreted in feces. However, some larvae change directly to filariform larvae and can autoinfect the host by penetrating the intestinal wall and again migrating to the lungs. This process of autoinfection, or hyperinfection, allows chronic infections to develop and can lead to a high worm burden, particularly in immunocompromised patients $[2,3]$.

Diagnosing Strongyloides requires a high index of suspicion. In our patient, the significant peripheral eosino- philia and elevated IgE level prompted stool testing for parasites. The initial stool sample was negative, highlighting the importance of testing numerous stool samples. The sensitivity of a single stool sample for Strongyloides is reported to be only $30 \%$, while the sensitivity of 7 samples can approach $100 \%$ [2].

The most common symptoms of strongyloidiasis are abdominal bloating, pain and diarrhea [4]. However, in a prospective study of patients in the Southeastern United States, $>70 \%$ of patients with strongyloidiasis also reported shortness of breath and wheezing [4]. Pulmonary manifestations range from bronchitis and asthma to acute respiratory failure and pulmonary fibrosis [4-10]. Interestingly, the most common radiographic findings in patients with pulmonary strongyloidiasis are diffuse alveolar opacities. Other radiographic findings include lobar and interstitial infiltrates, and fibrosis [2]. While PE are not commonly cited as a complication of strongyloidiasis, the prevalence of $\mathrm{PE}$ in patients with known pulmonary strongyloidiasis ranges from 3 to $40 \%[2,6]$. We reviewed existing literature and found only 5 published case reports on Strongyloides-induced PE [8, 11-14]. Of note, Strongyloides larvae were identified in pleural fluid in only one of these cases - an immunocompromised patient [11]. Similarly, we were not able to identify any larvae in the pleural fluid of our patient.

As the pulmonary manifestations of strongyloidiasis are often vague, there can be a delay in diagnosis. In one case series, the diagnosis of strongyloidiasis was delayed by a mean of 10 weeks in $70 \%$ of cases [6]. Furthermore, as the majority of patients with strongyloidiasis can present with shortness of breath and wheezing, a misdiagnosis of asthma or even allergic bronchopulmonary aspergillosis can occur. This can have fatal consequences as there have been at least 3 documented cases of patients with undiagnosed pulmonary strongyloidiasis expiring after receiving corticosteroids for presumed asthma [7, 8].

Our patient was treated with two courses of oral ivermectin. Two months after discharge, he reported significant improvement in his cough, shortness of breath and abdominal pain. Our laboratory evaluation revealed complete resolution of his peripheral eosinophilia, and by 4 months his IgE level had decreased by approximately $35 \%$. By 1 year, his Strongyloides antibody by EIA had decreased from 8.36 to 1.29 (reference range $<1$ ). A repeat CT scan of the chest performed 5 months after presenta- 
tion showed a persistent organized effusion, consistent with a fibrothorax, likely secondary to the chronic effusion. However, the airspace opacities previously identified in the right upper and middle lobes had resolved, which could have reflected the response to treatment for Strongyloides and/or methicillin-sensitive S. aureus pneumonia. While a transbronchial biopsy may have assisted in the diagnosis, this test has not been well validated as a means to detect Strongyloides, and its sensitivity and specificity are unknown [2]. Furthermore, because of our patient's cirrhosis-related splenomegaly and significant thrombocytopenia, the risks of this procedure were thought to outweigh the benefits. It is unfortunate that the cause of the patient's PE was not diagnosed sooner, as earlier treatment and drainage of this pleural fluid might have prevented the development of a fibrothorax. The patient was ultimately referred to a thoracic surgeon for definitive video-assisted thoracoscopic surgery, but declined.

In conclusion, as the pulmonary manifestations of strongyloidiasis can be exceedingly nonspecific, a high index of suspicion must be maintained in any patient with eosinophilic pulmonary disease from an endemic area. As mortality is between 50 and $80 \%$ in patients with disseminated infections [15], there should be considerable caution in the use of immunosuppressive corticosteroids.

\section{Acknowledgments}

We are grateful to this patient and his family for allowing us to share and publish his story.

\section{Financial Disclosure and Conflicts of Interest}

None of the authors listed report any conflicts of interest.

\section{Key Words}

Eosinophilia · Eosinophilic pleural effusion · Parasitic lung disease $\cdot$ Strongyloides stercoralis

\section{References}

1 Oba Y, Abu-Salah T: The prevalence and diagnostic significance of eosinophilic pleural effusions: a meta-analysis and systematic review. Respiration 2012;83:198-208.

-2 Mokhlesi B, Shulzhenko O, Garimella PS, et al: Pulmonary strongyloidiasis: the varied clinical presentations. Clin Pulm Med 2004; 11:6-13.

3 Levinson W: Nematodes; in Levinson W (ed): Review of Medical Microbiology and Immunology. New York, McGraw-Hill, 2010.

4 Berk SL VA: Clinical and epidemiologic features of strongyloidiasis: a prospective study in rural Tennessee. Arch Intern Med 1987; 147:1257-1261.

5 Bruno P, McAllister K, Matthews JI: Pulmonary strongyloidiasis. South Med J 1982;75: 363-365.
-6 Woodring JH, Halfhill H, Berger R, et al: Clinical and imaging features of pulmonary strongyloidiasis. South Med J 1996;89:10-19.

7 Boulware DR, Stauffer WM, Hendel-Paterson BR, et al: Maltreatment of Strongyloides infection: case series and worldwide physiciansin-training survey. Am J Med 2007;120:545548.

8 Higenbottam TW, Heard BE: Opportunistic pulmonary strongyloidiasis complicating asthma treated with steroids. Thorax 1976;31: 226-233.

$>9$ Cook GA, Rodríguez H, Silva H, et al: Adult respiratory distress secondary to strongyloidiasis. Chest 1987;92:1115-1116.

$>10$ Janssen R, Vlaminckx BJ, Seldenrijk CA, et al: Strongyloides stercoralis hyperinfection mimicking accelerated form of idiopathic pulmonary fibrosis. Lancet Infect Dis 2008;8: 456.
11 Win TT, Sitiasma H, Zeehaida M: Strongyloides stercoralis induced bilateral blood stained pleural effusion in patient with recurrent non-Hodgkin lymphoma. Trop Biomed 2011; 28:64-67.

12 Emad A: Exudative eosinophilic pleural effusion due to Strongyloides stercoralis in a diabetic man. South Med J 1999;92:58-60.

13 Goyal SB: Intestinal strongyloidiasis manifesting as eosinophilic pleural effusion. South Med J 1998;91:768-769.

14 Corrigan FL: A case of pulmonary strongyloidiasis. Br Med J 1949;ii:738.

15 Lam C, Tong M, Chan K, et al: Disseminated strongyloidiasis: a retrospective study of clinical course and outcome. Eur J Clin Microbiol Infect Dis 2006;25:14-18. 